A N N A L E S Annales de Bretagne et des Pays de l'Ouest

Anjou. Maine. Poitou-Charente. Touraine

113-3 | 2006

Prieurés et société au Moyen Âge

\title{
Cluny et les autres : l'exemple des prieurés
}

\section{Philippe Racinet}

\section{(2) OpenEdition}

Journals

Édition électronique

URL : http://journals.openedition.org/abpo/804

DOI : 10.4000/abpo.804

ISBN : 978-2-7535-1503-1

ISSN : 2108-6443

Éditeur

Presses universitaires de Rennes

Édition imprimée

Date de publication : 30 octobre 2006

Pagination : 203-221

ISBN : 978-2-7535-0405-9

ISSN : 0399-0826

Référence électronique

Philippe Racinet, «Cluny et les autres : l'exemple des prieurés », Annales de Bretagne et des Pays de l'Ouest [En ligne], 113-3 | 2006, mis en ligne le 30 octobre 2008, consulté le 19 avril 2019. URL : http:// journals.openedition.org/abpo/804; DOI : 10.4000/abpo.804 


\title{
Cluny et les autres : l'exemple des prieurés
}

\author{
Philippe RACINET \\ Professeur, Université de Picardie (Amiens) \\ LAHT, EA 3912
}

Envisager Cluny dans ses relations avec le monde extérieur, "les autres », c'est poser la question fondamentale de l'adaptation ou de la marginalisation de ses prieurés vis-à-vis d'une société en perpétuelle évolution, qui peut se décliner en fonction de trois thèmes : le besoin et l'envie des autres au moment de l'installation, en abordant le problème du don et les types d'implantation; l'opposition des autres sous la forme des litiges, des oppressions et des relations de mauvais voisinage, au moment où la société médiévale bascule dans la dépression; le secours des autres qui, lors de la même période de dépression, permet aux clunisiens de faire face.

\section{Le besoin et l'envie des autres}

\section{Les dons}

La puissance de Cluny repose avant tout sur les donations. Au Moyen Âge, le don est un mécanisme complexe et le transfert de propriété n'est pas un total transfert de possession, ce qui explique la mise en place de relations amicales (confirmations) ou conflictuelles (réclamations des héritiers) entre le monastère et le donateur ou sa famille. Le don renforce ainsi les liens entre les moines et leur environnement.

La complexité est encore accrue par la diversité des motivations. L'aspect religieux est le mieux connu et il est aisé de mettre en relation les dons, les prises d'habit, les prières et les inhumations. Les motifs économiques sont moins souvent avancés bien que fort intéressants. Le don peut être une sorte de placement : en 1219, Aumerus, clerc, donne à Joigny (diocèse de Sens) une dîme à condition qu'on lui paie, sa vie durant, autant de blé qu'elle vaudrait par amodiation ${ }^{1}$. Les motifs sociaux peuvent également être recherchés dans l'ampleur des relations personnelles qu'entretiennent prieurs et moines avec leur milieu d'origine, l'aristocratie, source princi-

1. Bibl. mun. de Joigny, ms. 18. 
pale des dons. Par ailleurs, la donation, élément de cohésion d'un groupe, est souvent une tradition familiale. Dans un sens proche, Barbara Rosenwein $^{2}$ insiste sur la raison principale du don qui serait de devenir un «voisin de Saint-Pierre ». Mais ce n'est qu'un aspect de ce fait social qu'on pourrait nommer "relations de bon voisinage». Si les laïcs cherchent à avoir de bons rapports avec les moines, l'inverse est également vrai car il y a là un potentiel de futures donations.

Un type particulier de donation, le don rétribué, a intéressé les historiens. Vers 1180, Vincent donne (dedi et concessi) une terre au prieuré de Longueville (diocèse de Rouen) et reçoit de caritate et de recognitione 25 livres angevines et 4 livres pour sa femme et ses héritiers ${ }^{3}$. Cette rétribution peut avoir plusieurs finalités : désamorcer d'éventuelles revendications de la part des héritiers, encourager les petits donateurs en leur accordant une sorte de pension viagère, marquer plus vigoureusement l'acte de donation lui-même.

La diminution progressive des actes de donation est une réalité à partir du XII ${ }^{\mathrm{e}}$ siècle mais elle aboutit rarement à une disparition complète des dons. La mettre au compte d'une baisse de la ferveur des donateurs potentiels constitue une schématisation excessive. En fait, c'est un signe de maturité car les donateurs privilégient naturellement les personnes ou les groupes qui en ont besoin!

\section{L'implantation}

Un monastère s'installe en fonction de données humaines. Cluny recherche les hommes et la géographie de son implantation se calque volontiers sur celle de l'habitat. Contrairement au courant cistercien, sorte d'érémitisme communautaire, le monachisme clunisien n'interpose pas un désert entre les populations et lui.

En milieu rural, il existe deux types d'implantation prieurale : le prieuré est en relation directe avec le village (à l'intérieur ou à la périphérie) ou il se dissocie de l'habitat villageois central. Dans $58 \%$ des cas français, le prieuré, presque toujours siège de la paroisse, est associé au village. La position centrale est la plus fréquente : à Rouy (diocèse de Nevers), l'église priorale et paroissiale est plantée au milieu du village (fig. 1). La position peut aussi être plus ou moins excentrée : le léger décentrement du prieuré de Cressonsacq (diocèse de Beauvais) s'explique par le fait qu'il a été attiré par le château (fig. 2). Lorsque l'implantation n'est pas en relation directe avec le village (20\%), le prieuré se trouve soit isolé dans la campagne, soit associé à un hameau monastique regroupant les familiers des moines. SaintNicolas d'Acy (diocèse de Senlis) ne se trouve pas dans le village de

2. RosenweIn, Barbara H., To Be the Neighbor of Saint Peter. The social Meaning of Cluny's Property. 909-1049, Ithaca-London, 1989.

3. Le Cacheux, P., Chartes du prieuré de Longueville, de l'ordre de Cluny, antérieures à 1204, Rouen, $1934, \mathrm{n}^{\circ}$ XXVI. 
Figure 1 - Rouy

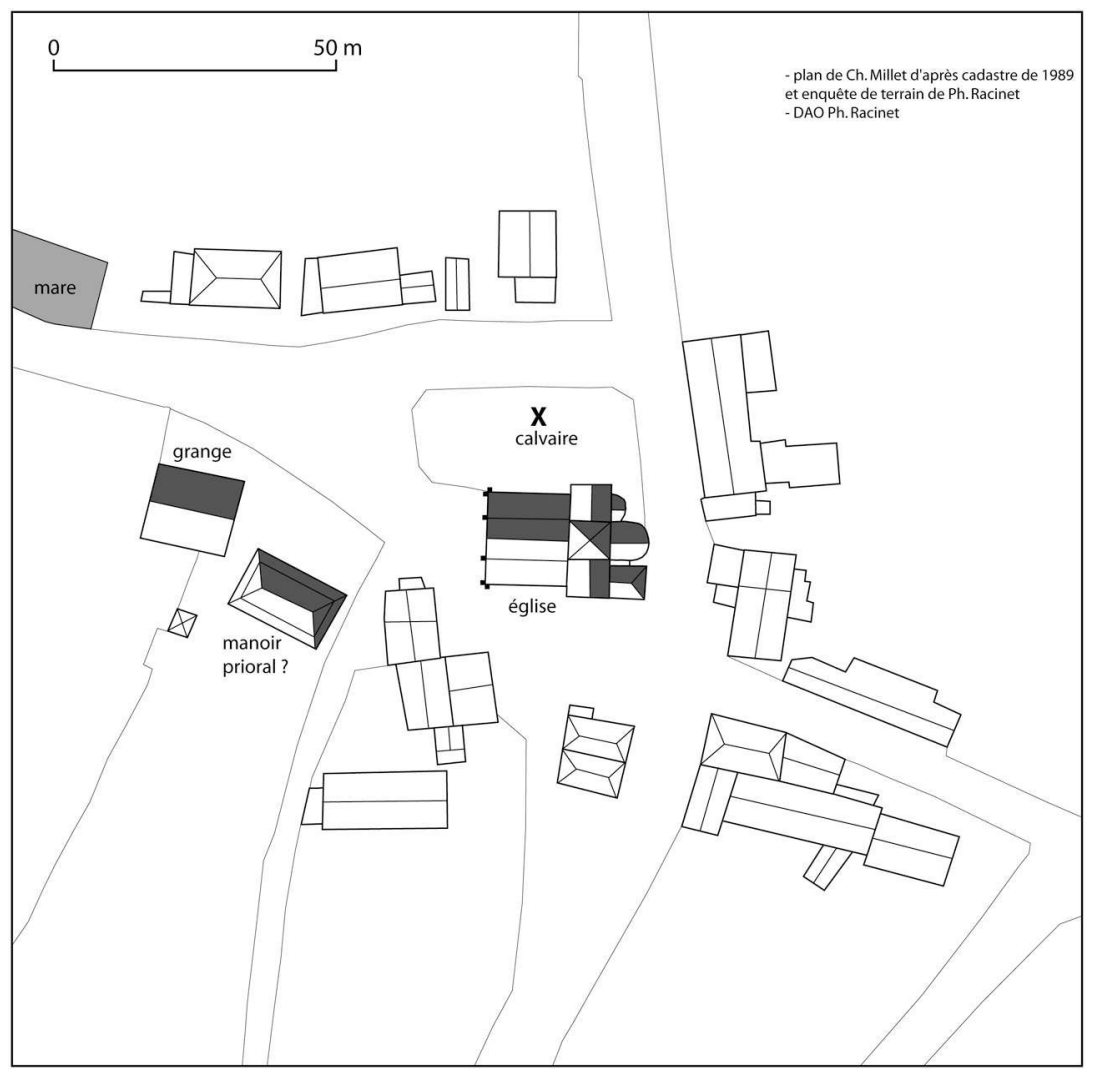

Courteuil mais il est intégré dans le hameau d'Acy qui, bien qu'antérieur au prieuré, lui est redevable de son extension en «village» monastique (fig. 3). La situation d'isolement peut aussi résulter d'une évolution : à Donzy-le-Pré (diocèse d'Auxerre), le village était groupé autour du prieuré puis il s'est déplacé, pendant la guerre de Cent Ans, pour se concentrer au pied du château. Il apparaît qu'en milieu rural, le prieuré ne se trouve jamais vraiment isolé de l'habitat. Il a très souvent été attiré par lui, il a presque toujours favorisé le peuplement et il a parfois créé un nouvel habitat.

Le nombre d'établissements installés en ville ou dans le faubourg d'une cité (22\%) montre que les clunisiens n'ont pas refusé une implantation en milieu urbain ${ }^{4}$. Dans certains cas, le prieuré n'a aucune action sur le développement urbain. Celui de Joigny (diocèse de Sens) s'implante en fonc-

4. RACINET, Philippe, «Prieurés clunisiens, bourgs et cités (milieu XI $\mathrm{e}^{\mathrm{e}}$-milieu XIII ${ }^{\mathrm{e}}$ siècle) », dans les Mélanges Pierre Desportes, Revue du Nord, t. LXXVIII, n 315, avril-juin 1996, p. 223-240. 
Philippe RACINET

Figure 2 - Cressonsacq

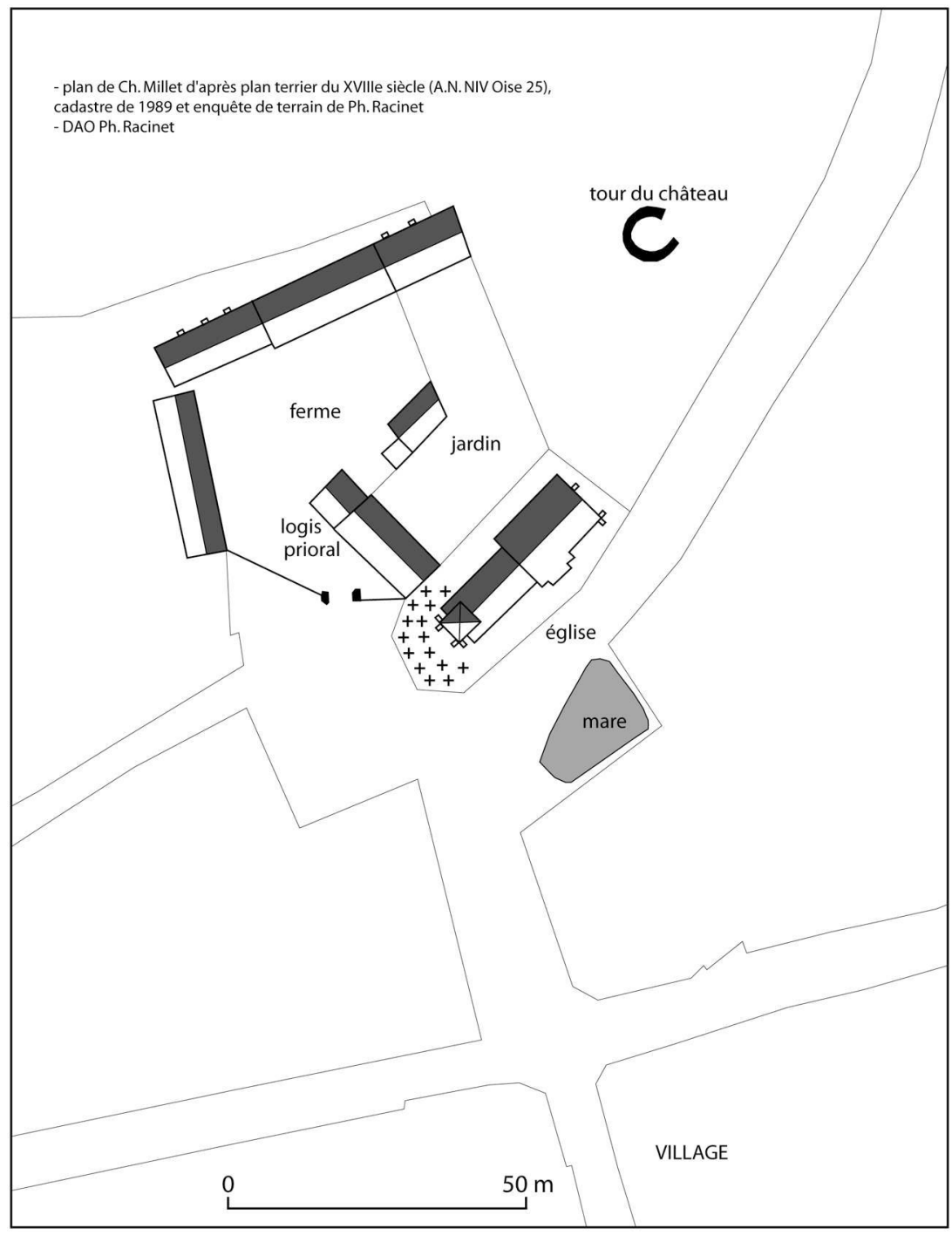

tion du château, vers l'est, alors que c'est à l'ouest que se développe le bourg (fig. 4). Cependant, il arrive fréquemment qu'un prieuré situé non loin d'une agglomération urbaine soit à l'origine d'un bourg. Établis au pied du château comtal, les moines de Nogent-le-Rotrou (diocèse de Chartres) ont su développer une puissance autonome en favorisant, avec le soutien des seigneurs, la constitution d'un bourg très actif disposant de nombreuses libertés (fig. 5). Ces prieurés urbains ou suburbains ont attiré des populations et créé des communautés bien individualisées qui jouè-

208 
Figure 3 - Saint-Nicolas-d'Acy

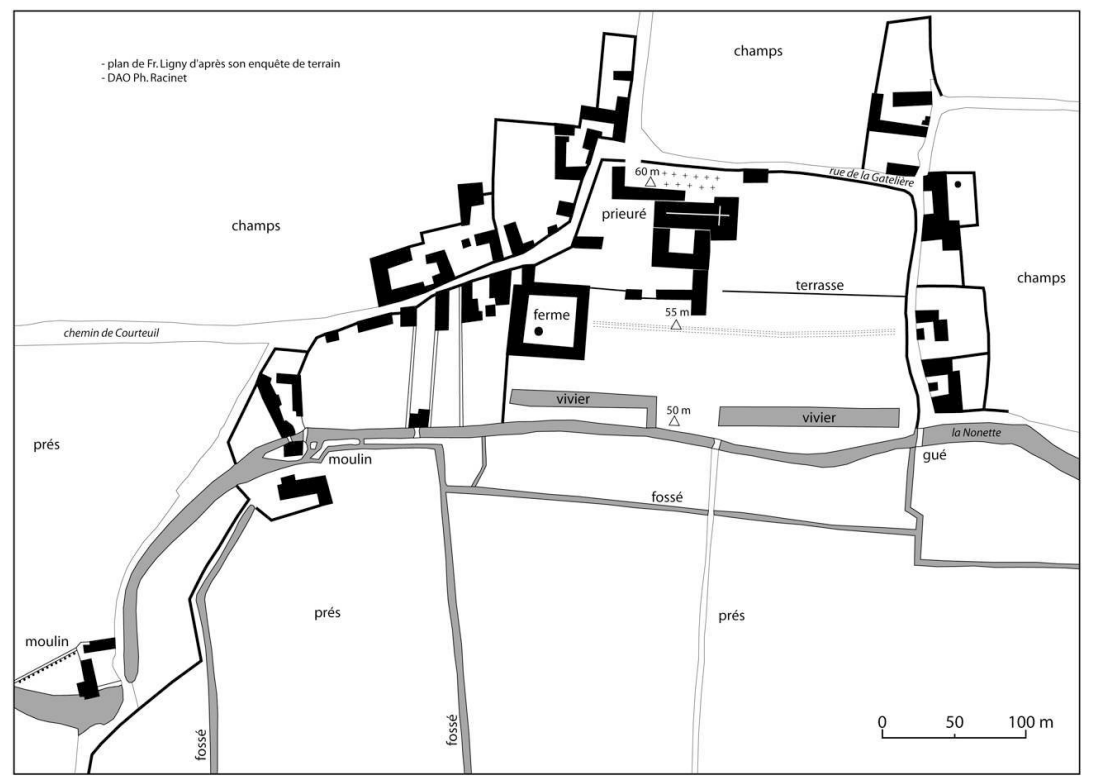

rent très vite un rôle aussi bien dans l'environnement du prieuré que dans la ville. Le pouvoir d'attraction du prieuré est fonction des privilèges qu'il peut drainer. Les fréquentes concessions du droit de foire furent aussi un élément capital dans le développement démographique des bourgs clunisiens. Dans certains cas exceptionnels, des monastères ont pu être à l'origine de villes. Le site même de La Charité-sur-Loire (diocèse de Nevers) a encouragé le développement urbain (fig. 6). Le témoin concret de ce développement fut l'édification en 1173 de la première enceinte de la ville 5 . Grâce en grande partie au pouvoir d'attraction du monastère, la cité poursuivit son essor et la diversification de ses activités, fondées sur l'exploitation d'un terroir rural riche du point de vue agricole et minier ainsi que sur le commerce interrégional entretenu par la présence du pont et du port.

La connexion de Cluny avec son environnement socio-économique entraîne une coïncidence logique entre l'implantation de ses prieurés et l'occupation humaine de l'espace médiéval. Le site d'un prieuré clunisien, comme sa situation, est fonction des hommes.

5. LeSPINASSE, René de, Cartulaire du prieuré de La Charité-sur-Loire, Nevers-Paris, 1887, $\mathrm{n}^{\circ}$ LXIV. 
Figure 4 - Joigny

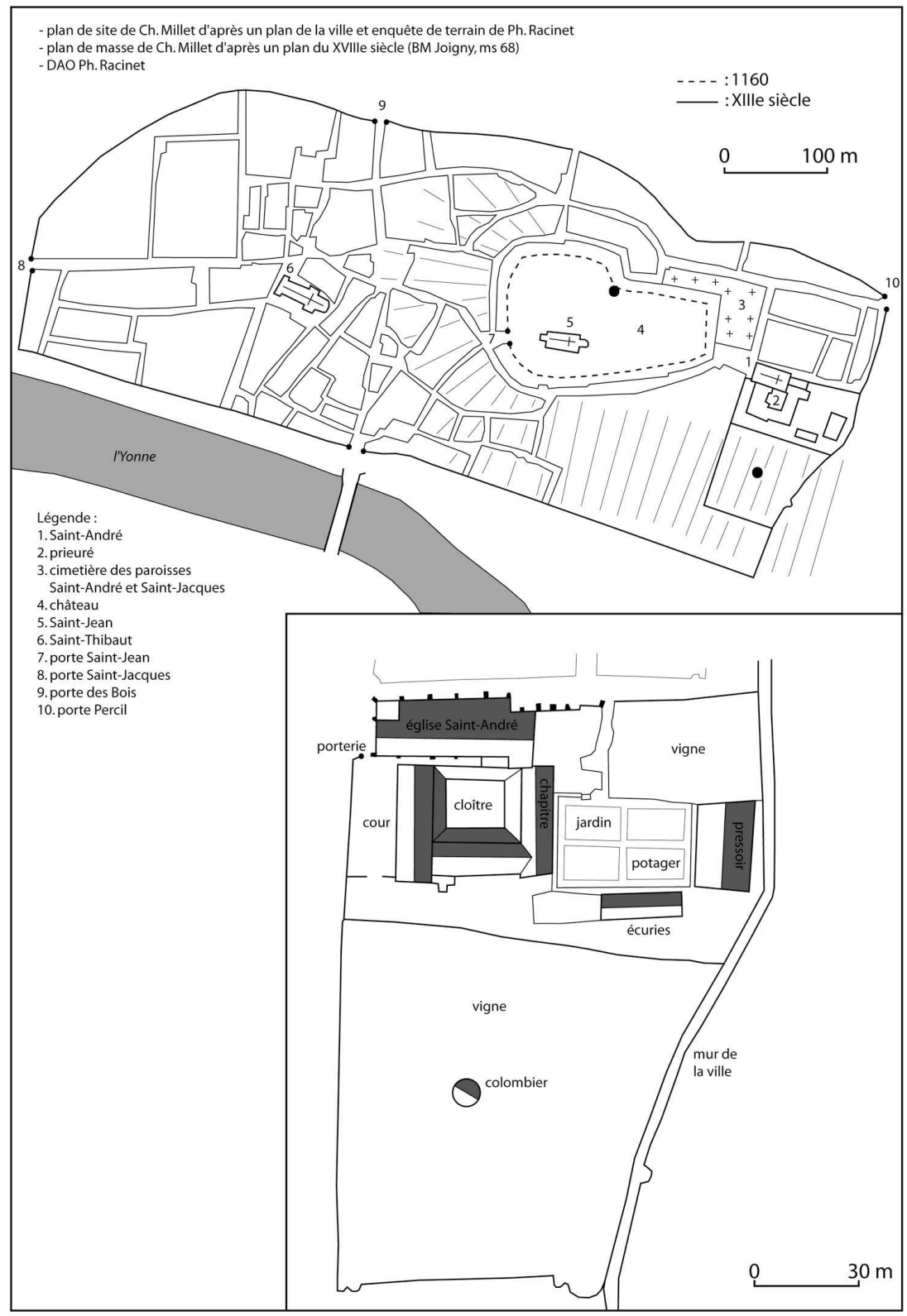


Figure 5 - Nogent-le-Rotrou

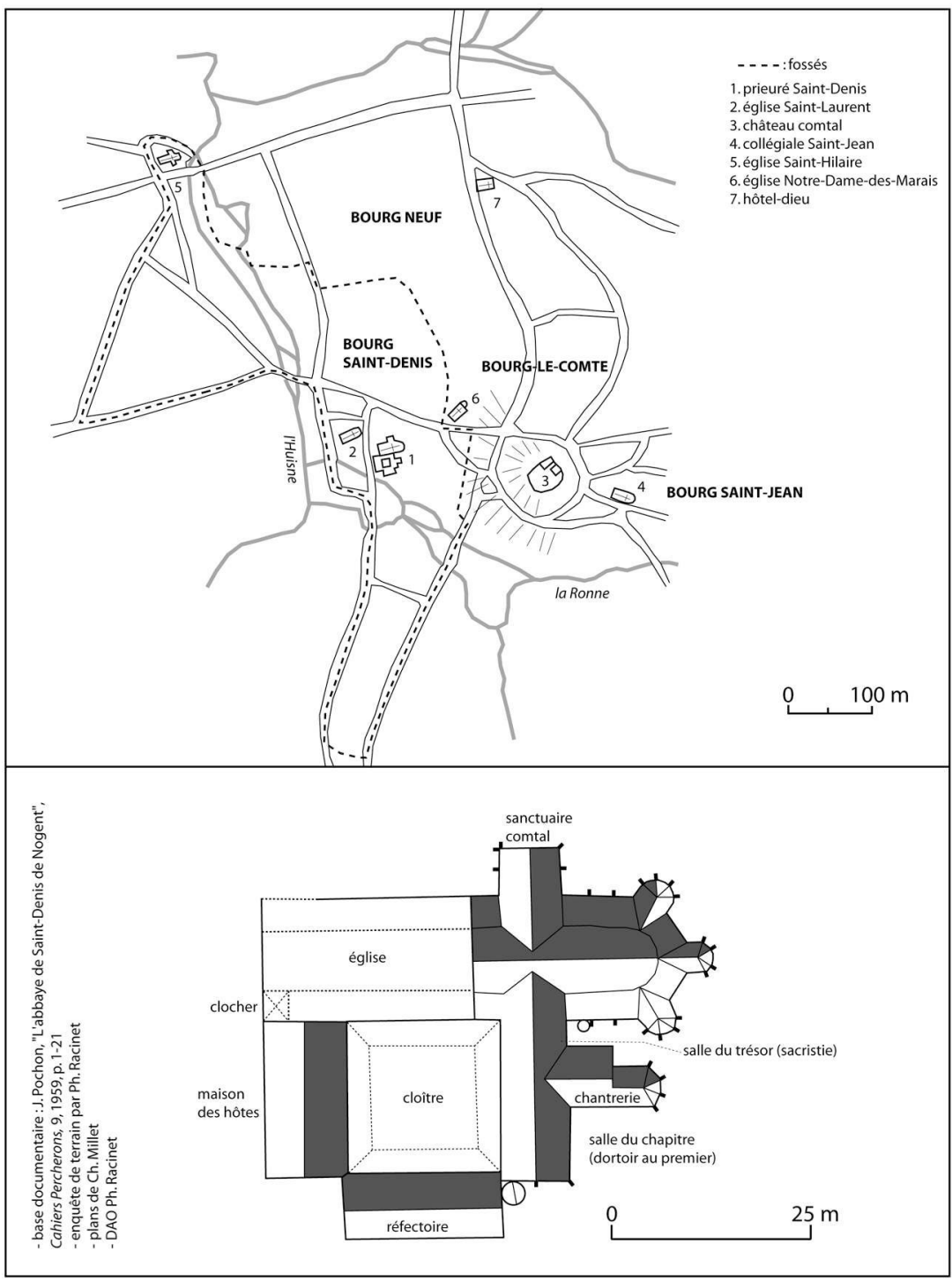

\section{L'opposition des autres}

$\mathrm{Au}$ XIII ${ }^{\mathrm{e}}$ siècle apparaissent des difficultés relationnelles, dans un environnement en mutation et en liaison avec une dégradation générale des conditions économiques. 
Figure 6 - La Charité-sur-Loire

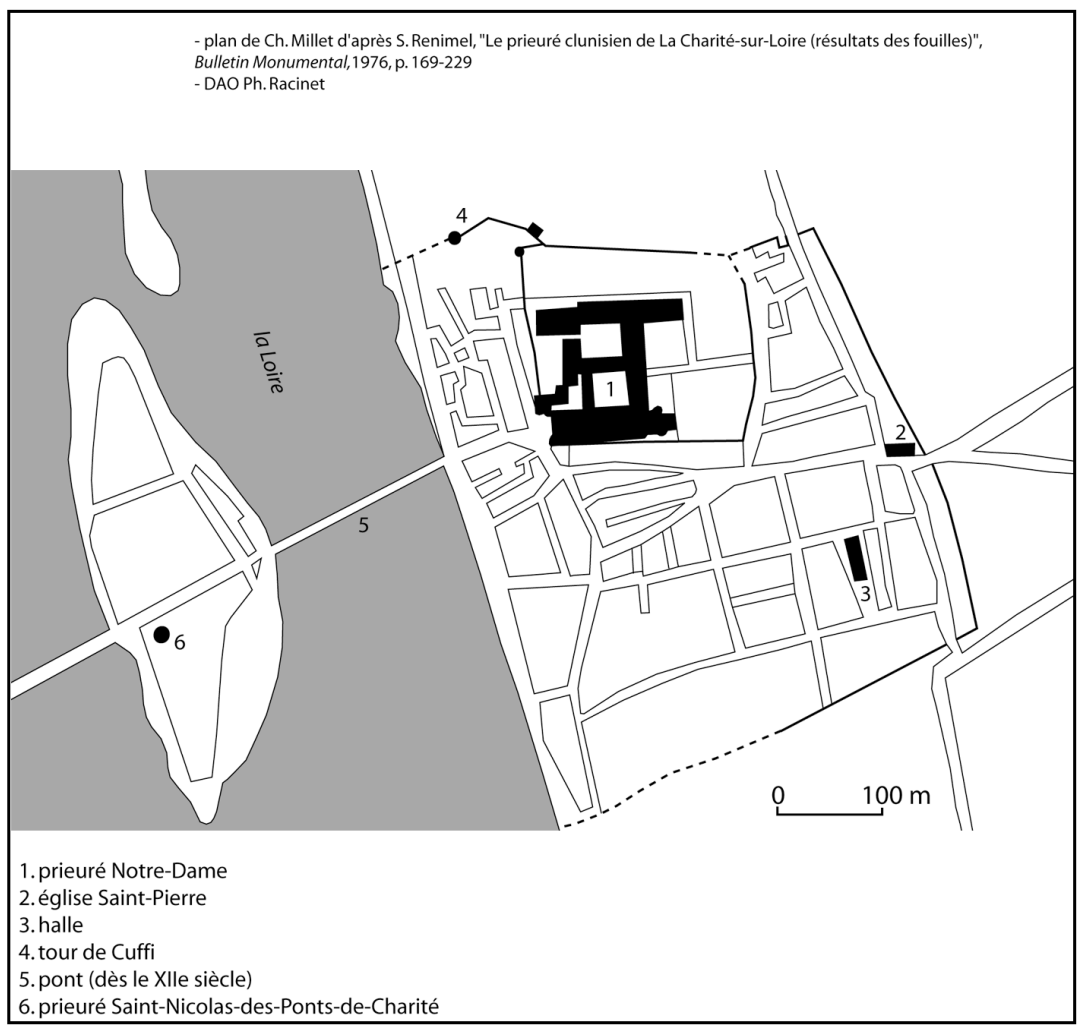

\section{Les monastères clunisiens}

\section{face aux transformations de l'environnement : le XIII siècle}

Les conflits avec les seigneurs, qui concernent les droits fiscaux et les juridictions, sont souvent d'une violence qui dénote une exacerbation des tensions. Lors d'une composition avec le seigneur Eudes de Beaujeu au sujet de la justice de Menetou (diocèse de Bourges), il est décidé en 1249 que le prieur de La Charité (diocèse de Nevers) recevra une première indemnité de 20 livres tournois pour les pillages commis par Eudes et ses hommes et une autre de 50 livres tournois pour coups et blessures portés contre le prieur de Menetou et son moine par Renaud Mareschal, probablement un agent du seigneur ${ }^{6}$. Outre ces violences d'intimidation ou de répression, on note aussi des tentatives de mainmise. Il ne faut pas, bien sûr, noircir le tableau et remarquer que les accords de bon voisinage restent les plus fréquents : en 1274, le prieur de Châteaudun (diocèse de

6. Lespinasse, René de, Cartulaire..., op. cit., $\mathrm{n}^{\circ} \mathrm{CX}$. 
Chartres) et le seigneur de Viviers procèdent, dans le calme et la bonne entente, au bornage de leurs terres ${ }^{7}$.

Si les mainmises épiscopales sur les prieurés apparaissent en Provence, on n'enregistre aucun cas semblable en France, mais les évêques y font de plus en plus opposition en tant qu'agents du roi. En 1293 et en 1298, plusieurs prieurs de la province de Reims se sont plaints à l'abbé de l'oppression de l'évêque d'Amiens qui, agissant pour le roi, a incarcéré un prieur, celui de Boves ${ }^{8}$. Le pouvoir princier devient, en effet, de plus en plus présent et les actes du Parlement de Paris montrent le début d'une véritable offensive contre les justices prieurales.

Les prieurés rencontrent aussi davantage de difficultés avec leurs hommes. Les religieux doivent tenir compte d'un environnement local qui ne cesse de s'affirmer, d'autant plus fortement dans les lieux d'implantation où les prieurés ont généré une importante communauté d'habitants. En 1249, un litige s'élève entre le prieur de Lihons-en-Santerre (diocèse d'Amiens) et les échevins, qui ont fait un sceau au détriment des droits du monastère ${ }^{9}$. Mais l'opposition peut être beaucoup plus violente. En 1263, un arrêt du Parlement condamne à des dommages et intérêts les habitants de Chaugnes qui ont maltraité et gardé en prison pendant un jour un moine de La Charité (diocèse de Nevers). Ce dernier avait trouvé un animal dans son bois et l'avait saisi car les habitants ne voulaient plus lui payer de droit de pâturage ${ }^{10}$.

À une plus grande échelle, le pouvoir municipal devient menaçant. Tout d'abord, il s'attaque à la justice des prieurés. Dès la première moitié du $\mathrm{XIII}^{\mathrm{e}}$ siècle, le prieuré Saint-Pierre Saint-Paul d'Abbeville (diocèse d'Amiens) rencontre des difficultés avec la commune du lieu sur cette question. Le développement urbain nécessite un premier accord, en 1254, pour la banlieue. En 1274, un autre accord sur divers points de juridiction a lieu avec le maire et les échevins et en 1292, le Parlement de Paris oblige le prieuré à reconnaître la ville comme seule détentrice de la haute et basse justice dans la vicomté de Saint-Pierre ${ }^{11}$. Ensuite, le pouvoir urbain attaque les prieurés dans leur rôle de patron ecclésiastique. On assiste à une sécularisation des paroisses urbaines, souvent en liaison avec le démembrement des cures primitives provoqué par le développement démographique. En fait, les moines pouvaient de moins en moins répondre aux nécessités religieuses et aux besoins d'assistance grandissants d'une population de plus en plus nombreuse.

7. Archives dép. d'Eure-et-Loir H 2661.

8. Dom Charvin, Gaston, Statuts, chapitres généraux et visites de l'ordre de Cluny, 9 vol., Paris, 1965-1979, t. II, p. 62 et 127.

9. Bibl. nat. de France, ms lat. 5460, $\mathrm{n}^{\circ} 12$.

10. Boutaric, Edgar, Actes du Parlement de Paris (1254-1328), 2 vol., Paris, 1863-1867, $\mathrm{n}^{\circ} 815$.

11. Collection privée du marquis de Belleval, Paris, charte de 1254 . Bibliothèque municipale d'Abbeville, Livre rouge, $\mathrm{f}^{\circ}$ XXVI et XIII. 
Bien que les moines sachent toujours défendre leurs droits, un environnement concurrentiel et menaçant se concrétise dans un contexte économique de plus en plus difficile. Les conflits opposent des puissances autonomes, reconnues et stabilisées. Personne ne conteste la légitimité des prieurés et jamais, il n'y eut de remise en cause de leur possession par Cluny. D'une manière générale, les relations se détériorent avec la grande dépression de la fin du Moyen Âge.

\section{Les prieurés comme enjeux des pouvoirs, oppressions et pressions au XIV siècle}

L'oppression doit être considérée comme un état de guerre. Elle provient soit d'un dérapage de la part de l'autorité publique qui outrepasse ses droits ou en revendique d'anciens (par exemple, la réquisition des biens d'Église pour fait de guerre), soit d'une usurpation de pouvoir de la part d'une autorité subalterne. Elle s'exprime de différentes façons. Le prieuré peut être occupé et gouverné par des séculiers. Il peut aussi s'agir d'une usurpation des biens ou des juridictions du prieuré. Cette situation est souvent liée à un environnement encombrant. En 1327, les liberi nobilis et potentis quondam viri domini Antalmi Aynaldi usurpent, comme leur père l'avait fait, d'importants revenus du prieuré de Saint-André-de-Rosans (diocèse de Gap) et le prieur ne peut pas résister à leur puissance qui s'exerce depuis dix ans ${ }^{12}$. L'oppression peut enfin se manifester, plus rarement, par des destructions matérielles, des attaques en règle ou encore des atteintes personnelles.

La pression politique provient nécessairement de l'autorité publique, royale ou princière. Si la garde royale est un moyen pour les moines de se protéger contre les oppressions seigneuriales, c'est aussi pour le roi l'assurance d'un contrôle certain. Dès fin du XIII ${ }^{\mathrm{e}}$ siècle, les souverains s'efforcent ainsi avec succès de récupérer la garde des prieurés clunisiens dans l'ensemble du comté de Nevers. Si cette protection est efficace, elle coûte cher. À partir de 1317, les chapitres généraux contiennent de longues mentions sur le roi "défenseur et gardien de l'ordre " mais les définiteurs concèdent des subsides ${ }^{13}$.

Si les monastères sont protégés, ils deviennent aussi des cibles pour l'autorité royale. Ce sont les justices prieurales qui sont le plus exposées. Les gens de justice du roi renforcent partout leur pression. La méthode la plus employée pour pénétrer les justices prieurales, le pariage, est douce. Cette pratique ancienne peut être totale ou ne concerner que certains droits, mais c'est toujours la porte ouverte à une intervention royale croissante. Plus ponctuellement, le roi profite de la conjoncture pour intervenir par le biais de l'arbitrage. Une autre façon de passer outre les justices prieurales fut l'utilisation des droits régaliens : en 1347, Philippe VI accorde

12. Dom Charvin, Gaston, Statuts, chapitres généraux..., op. cit., t. III, p. 49.

13. Ibidem, t. II, p. 424. 
rémission à Perrot Levesque qui avait dérobé du blé au serviteur du prieur de Bonnelles (diocèse de Chartres) et qui était poursuivi par ledit prieur, haut justicier du lieu ${ }^{14}$. En fait, les atteintes aux privilèges des monastères, que le roi était chargé de garder, étaient fréquentes et pouvaient provenir d'un excès de ses gens. Mais le souverain n'était pas sans procéder de la même manière quand il s'agissait de justice et de fiscalité. Oppression et pression peuvent, en effet, se manifester à travers la fiscalité.

À partir de 1340-1350, l'endettement des prieurés est presque exclusivement le fait des fiscalités pontificale et princière. La fiscalité pontificale n'est pas lourde en elle-même mais plusieurs faits expliquent qu'elle soit pesante à la fin du Moyen Âge. Elle est nouvelle dans sa régularité. Elle est rigide car sa base d'imposition n'est pas souvent révisée, si bien que la charge fiscale prend en compte avec beaucoup de retard l'évolution économique des prieurés. Elle s'ajoute à d'autres fiscalités anciennes, comme le cens à l'abbé, ou nouvelles, comme les impôts royaux ou princiers. En fait, les princes profitent d'un rapport de force nettement en leur faveur. Dans une société dominée par les problèmes séculiers, la recherche de protecteurs devient pour les moines une question cruciale. Obtenir la faveur et la protection du prince, c'est reconnaître son autorité. Même si la protection est souvent payante, elle est bien utile en ces temps de troubles. Le prince devient de plus en plus la référence ultime et le recours normal. Par ailleurs, la présence princière peut aussi offrir des avantages aux moines : en 1344, le comte de Savoie, qui tient le temporel du prieuré de Conzieu (diocèse de Belley) depuis au moins 1331, lègue une vigne et ses dépendances; du reste, prioratus et domos in pulcro statu dimisit ${ }^{15}$.

\section{Les relations de mauvais voisinage à la fin du Moyen Âge}

Les oppositions représentent un autre aspect du climat de tension et s'expriment tout d'abord par les procès. Entre 1291 et 1350, les mentions de débats sont deux fois plus nombreuses que pour l'ensemble de la période comprise entre 1260 et 1490. Les oppositions se traduisent aussi par une violence notoire. En 1300, un moine de Nogent-le-Rotrou (diocèse de Chartres) est assassiné par des nobles ${ }^{16}$. Violence physique mais aussi matérielle : en 1315, le prieur de Cappy (diocèse de Noyon) fait appel au roi au sujet de la destruction d'un moulin perpétrée par plusieurs complices identifiés ${ }^{17}$.

Les seigneurs laïques se retrouvent souvent dans les procès mais ce fait n'est pas nouveau. De même, les litiges avec les curés au sujet des revenus paroissiaux ne sont que des oppositions localisées et bien connues avant. En fait, ce sont les habitants qui constituent sûrement la catégorie d'op-

14. Arch. nat., série JJ 76, n 46.

15. Dom Charvin, Gaston, Statuts, chapitres généraux..., op. cit., t. III, p. 375.

16. Ibidem, t. II, p. 154-155.

17. Arch. nat., série S $1412, \mathrm{n}^{\circ} 3$. 
posants la plus dangereuse dans la mesure où ces conflits ternissent nécessairement l'image du prieuré. Les communautés d'habitants sont particulièrement actives en Provence et les moines sont souvent obligés de composer. En 1302, une transaction " pour la paix et la concorde » est passée entre le prieur de Pont-Saint-Esprit (diocèse d'Uzès) et les habitants de Tulette (diocèse de Vaison) au sujet des servitudes et des redevances. Les droits du prieur sont sauvegardés mais on s'achemine vers une indépendance de plus en plus grande de la communauté ${ }^{18}$.

Avec la guerre, l'opposition des habitants est d'autant plus fortement ressentie que les circonstances obligent les moines à vivre davantage dans le siècle. Elle peut s'exprimer sous la forme d'une mauvaise volonté comme l'enseignent les comptes rendus de visite relatifs aux réparations à effectuer dans les églises paroissiales. Les contentieux sont nombreux et souvent difficiles à résoudre étant donné l'incertitude concernant la responsabilité de chaque partie. La pression des habitants s'exprime aussi très couramment à travers la rumeur publique. C'est une façon de faire éclater les scandales et peut-être de corriger les abus. Quand la voix populaire s'exprime clairement sous la forme de plaintes, son efficacité ne fait aucun doute. La crainte de l'opinion publique devient presque une obsession pour les autorités centrales. Le mécanisme, parfaitement compris, est évoqué en 1409 à propos de la maladie du sacristain de Lurcy-le-Bourg (diocèse de Nevers) : Sacrista dicti loci fuit alias diffamatus de morbo lepre - et adhuc suspicatur - contra eum fama currente - et possit verti in damnum religiosis cum ipso conversantibus ${ }^{19}$. Dans leurs prescriptions, visiteurs comme définiteurs prennent en compte l'opinion du milieu dans lequel le prieuré concerné se trouve placé.

Toutes ces oppressions, pressions et oppositions sont-elles le signe d'une désaffection, au moins provisoire, des populations? Peut-être, dans la mesure où les donations enregistrées pour la période 1291-1330 sont deux fois moins nombreuses que celles de la période précédente (12511290). Mais il ne faut pas oublier que la violence se manifeste désormais dans la vie quotidienne. Entre 1313 et 1336, les moines de Saint-Sébastiende-Cordiac (diocèse de Die) ne cessent de se plaindre des oppressions du seigneur de Morges ${ }^{20}$. Celui-ci agit-il par haine des clunisiens ? Sûrement pas puisqu'il a un fils dans l'ordre!

Ainsi, les tensions existent, la pression est réelle et peut agir négativement sur l'évolution des prieurés. Mais il ne faut rien exagérer : le prieuré n'est pas entouré d'un monde hostile avec lequel il n'a plus de contact. Les moines gardent des rapports privilégiés avec la noblesse ou la bourgeoisie, et le monde rural populaire est loin d'être systématiquement hostile. Nous pouvons citer deux exemples précis : en 1389, les villageois s'asso-

18. Malbois, Émile, « Notice historique sur Tulette », Bulletin de la Société archéologique de la Drôme, 1926, p. 17-53.

19. Dom Charvin, Gaston, Statuts, chapitres généraux..., op. cit., t. V, p. 5.

20. Ibidem, t. II, p. 342 et t. III, p. 234. 
cient aux moines de Saint-Thibaud (diocèse de Châlons) pour déclarer aux visiteurs que le prieur du lieu et son socius manquent de vin, de blé et de pitance; en 1404, le prieur de La Charité-sur-Loire (diocèse de Nevers) envoie au chapitre général deux notables locaux comme procureurs ${ }^{21}$.

\section{La fidélité et le secours des autres à la fin du Moyen Âge}

Si la présence de Cluny, comme celle des autres ordres monastiques, au sein des grandes instances intellectuelles et politiques du temps s'est réduite à la fin du Moyen Âge, le fort enracinement local de ses moines lui permet de conserver un réel rayonnement qui s'exprime à travers les relations qu'entretiennent ses maisons avec leur environnement.

\section{Le maintien d'un rayonnement}

Les nécrologes montrent que le milieu clunisien continue d'être parfaitement inséré dans le paysage social de son temps et les demandes d'inhumations, toujours nombreuses, rappellent que les églises prieurales conservent leur pouvoir d'attraction. Pour des raisons à la fois financières, religieuses et sociales, les moines accordent toujours une grande importance à ce rôle funéraire; les futurs défunts également puisqu'ils n'hésitent pas, parfois, à faire des chantages à la sépulture. En 1401, le seigneur de Morges, qui se dit «fondateur» de Saint-Sébastien-de-Cordiac (diocèse de Die), menace d'instituer sa sépulture ailleurs et « de reprendre tous les droits qu'il a donnés ${ }^{22}$ ». Simples paroles en fait, car les laïcs n'avaient pas la possibilité psychologique de jouer avec l'au-delà en ces temps de mort rapide. Les relations avec l'aristocratie locale amènent le prieuré à jouer un véritable rôle de sanctuaire quand les membres de cette noblesse descendent de la lignée fondatrice ou s'identifient à elle. Les fouilles du site de Nanteuil-le-Haudouin (diocèse de Meaux) révèlent que l'église prieurale a eu cette fonction pour la famille seigneuriale entre le XII ${ }^{\mathrm{e}}$ et le XVII ${ }^{\mathrm{e}}$ siècle ${ }^{23}$. Les clunisiens, se fondant sur la pérennité de leur tradition, profitèrent largement des angoisses du temps face à la mort. C'est bien par l'intermédiaire de la fonction funéraire (inhumations et anniversaires) que s'exprime pleinement la fidélité des familles fondatrices. En 1353, Pierre de Luyrieu confirme toutes les donations faites au prieuré de Talissieu (diocèse de Genève) par ses ancêtres et, en échange d'une augmentation de dotation, le prieur s'engage à célébrer trois messes par semaine pour les défunts de cette famille dans la chapelle de la Vierge, sanctuaire familial. Deux ans plus tard, Pierre exprime, par testament, sa volonté d'être inhumé dans la chapelle; l'acte précise que, de toute antiquité, les Luyrieu ont été regardés

21. Ibid., t. IV, p. 267 et $495-496$.

22. Ibid., t. IV, p. 453.

23. RACINET, Philippe, «Approche pluridisciplinaire : le prieuré de Nanteuil-le-Haudouin du XI ${ }^{\mathrm{e}}$ au XVIII ${ }^{\mathrm{e}}$ siècle (Oise) ", dans Abbayes et prieurés. Communautés religieuses en Île-deFrance, Paris et Île-de-France, Mémoires, t. 48, 1997, p. 79-99. 
comme fondateurs du prieuré ${ }^{24}$. Ces liens très forts n'impliquent pas nécessairement une mainmise sur l'établissement. Il n'y eut aucun Luyrieu parmi les prieurs de Talissieu.

Les donations constituent le témoignage concret de la fidélité des milieux en contact avec le monde clunisien. Elles se maintiennent tout au long du XIV ${ }^{\mathrm{e}}$ siècle à leur niveau de la fin du XIII ${ }^{\mathrm{e}}$ siècle. Il ne s'agit pas d'actes fondés sur l'usage mais bien de donations volontaires et motivées : Aoude, en 1409, donne à Saint-André-de-Rosans (diocèse de Gap) 12 florins d'or car elle est « satisfaite de l'office divin qui se fait chaque jour avec dévotion dans le monastère ${ }^{25}$ ». À La Charité-sur-Loire (diocèse de Nevers), les 39 actes de donations, recensés entre 1350 et 1418, proviennent en nombre sensiblement égal de l'aristocratie locale et de la bourgeoisie. Il s'agit essentiellement de fondations d'anniversaires accompagnées parfois de demandes de sépulture près de parents déjà enterrés dans l'église. Les textes prouvent la réelle sincérité de ces démarches et montrent combien le prieuré clunisien continue d'exercer son attrait spirituel au milieu de la tourmente. En 1407, le doyen de Nogent-le-Rotrou (diocèse de Chartres) notifie que Guillaume le Taillandier, bourgeois, et sa femme donnent une métairie «a peurement de devotion... pour le salut de leurs ames». Les conditions sont précises : jouissance viagère du bien concédé, association aux prières des moines et célébration de quatre anniversaires ${ }^{26}$.

Toutes ces manifestations de bienveillance sont directement liées à la confiance accordée par le peuple aux moines clunisiens. Le prieuré avait sa place et conservait ses rôles. Rôle d'abord religieux dans le sens le plus général de ce terme; en 1350, un visiteur de Cluny, déclare que les habitants d'Encre (diocèse d'Amiens) sont venus le voir pour se plaindre de la disparition d'une messe et demander que cet office soit célébré tous les matins par le prieur et les religieux ${ }^{27}$. Rôle d'assistance au sens large ensuite; en 1389, le prieur de Margerie-Hancourt (diocèse de Troyes) multum sit oneratus... per multitudinem hospitum ${ }^{28}$... Cette confiance, s'appuyant sur une estime réciproque, va s'avérer utile lorsque le ralentissement des troubles permettra d'intensifier les efforts de reconstruction.

\section{Etre moine au temps de l'humanisme}

André Vauchez a montré qu'aux XIV et $\mathrm{XV}^{\mathrm{e}}$ siècles, un clivage se produit entre les dévots et les mystiques, d'une part, et la masse attachée aux pratiques (individuelles et collectives) d'ordre avant tout utilitaire, d'autre part $^{29}$. En maintenant une vie monastique convenable et traditionnelle, les

24. Arch. dép. de l'Ain, H 83bis.

25. Arch. dép. de l'Isère, 3E 282 , fo 87.

26. Sounancé, Charles de, et MÉTAIS, Charles, Saint-Denis de Nogent-le-Rotrou, histoire et cartulaire, Vannes, $1899, \mathrm{n}^{\circ} \mathrm{CXXXIII}$.

27. Bibliothèque municipale d'Amiens, ms. 1264.

28. Dom Charvin, Gaston, Statuts, chapitres généraux..., op. cit., t. IV, p. 267.

29. VAuCHEZ, André, Les laïcs au Moyen Âge, Paris, 1987, p. 289-291. 
clunisiens ont su conserver une influence religieuse dans la société. La prière continue d'être le centre de la vie religieuse des moines. Tout au long de la fin du Moyen Âge, les définiteurs rappellent que la fonction première d'un prieuré est liturgique. Ce rôle, toujours revendiqué par les moines à l'époque moderne, est aussi celui que continue de leur assigner l'entourage, seigneurial en particulier. Gardiens d'une tradition liturgique, les clunisiens savent aussi préserver les points de fixation de leur rayonnement religieux : les reliques et les pratiques funéraires. Les moines entretiennent tout d'abord le pôle d'attraction que constituent des reliques conservées avec soin. Objets de sollicitude, elles ponctuent toujours les principaux événements des grands prieurés au XVI ${ }^{\mathrm{e}}$ siècle.

Les églises clunisiennes continuent aussi d'attirer les morts. Les inhumations concernent non seulement l'aristocratie locale mais aussi le clergé séculier, et s'accompagnent d'une recherche consciente des prières clunisiennes comme le montre cet exemple : en 1532, Jean de Baugy, contrôleur du grenier à sel de La Charité-sur-Loire (diocèse de Nevers), avait légué par testament 31 . de rente, à la charge de dire tous les dimanches un de Profundis pour le repos de son âme. Mais les religieux ayant refusé d'accepter la fondation parce que la rente était trop faible, sa fille, veuve du bailli de La Charité, l'augmente ${ }^{30}$.

Le sérieux religieux de nombreux moines contribue à maintenir l'influence locale des prieurés. Vers 1530-1532, frère Lambert Hottement, ancien prieur de Saint-Maurice de Senlis (monastère de chanoines réguliers), fréquente régulièrement le prieuré de Saint-Nicolas d'Acy (diocèse de Senlis) «pour ce que Dieu y est bien servy et l'église et la maison en bon ordre et observance régulière ». Ce rayonnement s'explique par une vie monastique correcte, « honnête » disent parfois les textes. En 1557, maître Jacques Dumoulin, procureur au Châtelet de Paris, donne au prieuré de Saint-Nicolas d'Acy une ferme, qu'il avait achetée 3672 livres tournois. Il explique ainsi son geste généreux : «[...] informé et adverty de la bonne vie et régulière observance des vénérables religieux, prieur et couvent de Sainct-Nicolas d'Acy-lès-Senlis... et du beau et solempnel service qui ordinairement et journellement se faict et cellèbre par iceulx religieux et prieur en l'église d'iceluy prieuré, sur le grant autel de laquelle église repose et est continuellement et très révérentement gardé le très précieux corps de Nostre Sauveur ${ }^{31} \ldots »$

Les 11 et 12 janvier 1568, une information est donnée "pour raison de la démolition et ruine de la maison du prieuré » de Grandchamp (diocèse de Meaux) par les Huguenots du capitaine de la Forest (1567). Dix-neuf témoins, habitant le village, déposent à la demande du prieur, contre les auteurs du pillage devant la justice royale ${ }^{32}$. Ces dépositions révèlent plu-

30. Histoire Cronologique du prieuré de La Charité-sur-Loyre, ordre de Cluny, Association «Les Amis de La Charité-sur-Loire », 1991, p. 100.

31. Arch. dép. de l'Oise, H 2586 et 2646.

32. Bobard, L., Pillage du prieuré de Grandchamp en 1567, Fontainebleau, 1900. 
sieurs éléments capitaux qui prouvent la force du monachisme clunisien en ce troisième quart du $\mathrm{XVl}^{\mathrm{e}}$ siècle :

- le rayonnement de la maison puisque les témoins «savent bien ce qu'ils avancent, parce qu'ils ont chanté ou fréquenté le prieuré, et ont été par plusieurs fois audict lieu »;

- l'excellente tenue des religieux qui « se sont toujours bien et notablement gouvernés sans estre notés ni accusés d'aucun cas de blasme ou reproches »;

- le maintien du rôle religieux du prieuré qui répond à la demande de prières liturgiques du peuple chrétien : «le service divin et de Nostre Mère Saincte Eglise est scélébrée avec exactitude »;

- le maintien du rôle social du prieuré puisque «audict Grand Champt étoit fait aulmosnes aux pauvres y survenant».

Si le déclin des valeurs monastiques dans l'Église de France et auprès des populations doit ainsi être relativisé, la question des rapports avec les valeurs humanistes peut être posée. La nouvelle mentalité s'est parfois opposée au monachisme tout en influençant la manière d'être et de vivre des religieux. Le moine clunisien au XVI ${ }^{\mathrm{e}}$ siècle est-il d'un nouveau genre? C'est un moine instruit ou qui cherche à l'être, et l'instruction est considérée comme un besoin. L'organe central a encouragé les études et les prieurs docteurs deviennent assez nombreux, tant à la tête des monastères conventuels que dans les petits prieurés. Même s'il ne fait donc aucun doute que les collèges clunisiens attirent de jeunes écoliers d'autres congrégations, les comptes rendus des chapitres généraux montrent que cette institution paraît à certains contemporains anachronique au sein du nouveau monde intellectuel du Xvi ${ }^{\mathrm{e}}$ siècle $^{33}$. Moines contre laïcs, nouveaux conquérants de la culture! Les définiteurs évoquent cette question en 1526 et en $1527^{34}$. Les moines font l'objet des plaisanteries des gens du siècle ...quinimo ludibrio esse religiosos secularibus tanquam mortuos seculo, indeque nomen religionis habere contemptui... La réflexion est profonde et pose le problème du rôle des moines dans le monde de la Renaissance. En fait, après l'effervescence intellectuelle de la première moitié du XVI ${ }^{\mathrm{e}}$ siècle et une fois les espoirs humanistes rangés au placard, lorsque les haines religieuses commencèrent à l'emporter, les collèges clunisiens retrouvèrent une utilité publique : ils se devaient d'être les bastions d'une vie religieuse traditionnelle et catholique ${ }^{35}$.

33. RACINET, Philippe, «Les collèges clunisiens, créations tardives et espoirs déçus (fin $\mathrm{XIII}^{\mathrm{e}}$-fin XVI ${ }^{\mathrm{e}}$ siècle) ", dans Voyages en histoire. Mélanges offerts à Paul Gerbod, Annales littéraires de l'Université de Besançon, $\mathrm{n}^{\circ}$ 550, Séries historiques n 9, Besançon-Paris, 1995 , p. $25-41$.

34. Dom Charvin, Gaston, Statuts, chapitres généraux..., op. cit., t. VI, p. 74 et 77.

35. RACINET, Philippe, "Marguerite de Navarre et le monde monastique du XVI ${ }^{\mathrm{e}}$ siècle : autour de la Nouvelle XXII de l'Heptameron", dans Fontevraud. Histoire. Archéologie, Comité d'Histoire fontevriste, $\mathrm{n}^{\circ} 2,1993, \mathrm{p}$. 55-66. 
Tradition et adaptation caractérisent la vie clunisienne dans le sens d'une fidélité à une manière d'être et de vivre. Adaptation face à un passé récent troublé et agressif. Adaptation également à de nouvelles conditions économiques, sociales et psychologiques. Au Xvl ${ }^{\mathrm{e}}$ siècle, le religieux clunisien est-il un homme «nouveau »? La réponse est non si l'on voit en cet adjectif une négation de son histoire. La réponse est oui dans la mesure où ce moine est un «homme de son temps». Mais comment aurait-il pu en être autrement?

Le maintien de l'option traditionnelle, le retour de la discipline et la restructuration économique, qui ont touché de nombreux établissements, montrent que le monachisme ancien a survécu à la dépression de la fin du Moyen Âge, mais il s'est adapté en s'accommodant d'une société où il est réduit à la portion congrue, par rapport à celle qu'il avait connue entre le $\mathrm{VIII}^{\mathrm{e}}$ et le $\mathrm{XII}^{\mathrm{e}}$ siècle. La présence des établissements clunisiens dans la société de la fin du Moyen Âge est un fait. Les témoignages des laïcs, donateurs ou non, à Grandchamp et ailleurs, montrent qu'il n'y a pas eu rejet de cette forme de vie religieuse. Des jeunes comme des adultes, de toutes origines, continuent d'être attirés par Cluny et ses maisons. Il est toujours possible de s'interroger sur les raisons qui poussent les hommes à devenir moines de Cluny à la fin du Moyen Âge et à l'époque moderne. Est-ce l'attrait spirituel ou la recherche d'une sécurité matérielle? Mais même dans cette seconde hypothèse, Cluny ne garde-t-il pas son utilité sociale? Du reste, si une institution surmonte de longues et multiples difficultés, c'est qu'elle a des raisons de vivre.

\section{RÉSUMÉ}

L'abbaye de Cluny est demeurée ouverte au monde tout au long du Moyen Âge. Ses prieurés, fondés grâce à de multiples dons, sont installés dans les villages et dans les villes. Avec les difficultés du XIII ${ }^{\mathrm{e}}$ siècle, ces derniers doivent affronter litiges et mauvais voisinage, mais à la fin du Moyen Âge, les clunisiens font face aux crises et secourent les autres. Tout au long des siècles, ils ont su faire preuve d'adaptation.

\section{ABSTRACT}

The abbey of Cluny remained open to the world all along the Middle Ages. His priories founded thanks to gifts are installed in the villages and towns. With the difficulties of the thirteenth Century they have to bear up against contests and bad neighbourhood at the end of the Middle Ages clunisians face up to crisis and assist others. All along centuries, they give proof of adaptation. 



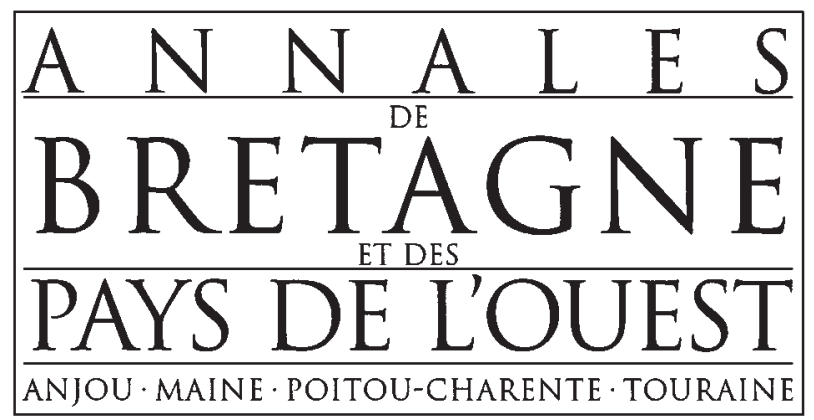

\section{ABONNEMENT}

FRANCE, 4 numéros : 32 e - ÉTRANGER, 4 numéros : 40 e (Franco de port)

ADRESSE D'EXPÉDITION

(pour toute commande)

Nom

Prénom

Organisme

Adresse

Code postal

Ville

Pays

Ce bon de commande accompagné d'un chèque à l'ordre de Agent comptable de l'Université Rennes 2 est à envoyer à l'adresse suivante :

Presses Universitaires De Rennes Campus de La Harpe 2 rue du Doyen-Denis-Leroy 35044 RENNES Cedex 


\section{QuelQues RÈGLes DE PRÉSENTATION DES TEXTES À L'USAGE DES AUTEURS}

1 - Indiquer sous le titre de l'article la qualité et l'adresse institutionnelle de l'auteur Ex. : Xxxx Xxxxxx, maître de conférences en histoire contemporaine, université de X., groupe de recherches de rattachement.

2 - Remplacer le plus possible les majuscules (sauf pour les lettres initiales des noms propres) par des petites capitales. Ceci dans les titres, les sigles, les noms d'auteurs... Indiquer aussi les siècles en petites capitales : $\mathrm{XV}^{\mathrm{e}}$ siècle et non XVe siècle.

Accentuer les majuscules.

3 - Abréviations : en user le moins possible

Pas d'abréviations pour le nom des revues, surtout pour les revues locales qui ne sont connues que des locaux.

Pour les dépôts d'archives :

- ne pas mettre ADIV ou AD 35 mais : Arch. dép. d'llle-et-Vilaine, du Morbihan...;

- préférer Arch. nat. à AN;

- mettre les noms des bibliothèques en entier.

Développer les sigles entre parenthèses lors de leur première occurrence.

4 - Références bibliographiques

- mettre toujours et partout les prénoms et les noms (ne pas abréger le prénom par une initiale);

- utiliser les petites capitales pour le nom propre et non les majuscules, utiliser les minuscules pour le prénom; les séparer par des virgules.

Nom, Prénom, Titre de l'ouvrage en italique, Lieu d'édition, éditeur, «collection » (éventuellement), année, pages.

Une bibliographie peut être donnée en fin d'article si cela est nécessaire.

5 - Forme des notes

Préférer la forme traditionnelle (référence biblio. développée lors de sa première occurrence, abrégée ensuite) à la forme anglo-saxonne (nom, date, page).

6 - Titres et sous-titres

Il est préférable de s'en tenir à deux niveaux. Mais si cela s'avère nécessaire afin de ne pas obtenir de trop longues pages trop compactes, un troisième niveau d'intertitres peut être utilisé.

7 - Cartes et documents photographiques

- Les cartes au trait devront être d'une EXCELLENTE QUALITÉ GRAPHIQUE sans collage hasardeux ni légende manuscrite.

- Il est toujours préférable de FOURNIR LES ORIGINAUX, sinon, un fichier informatique au format .EPS pour les cartes et plans ou .TIF pour les photographies. en aucun cas les images seront intégrées au fichier word seulement : IL FAUT ÉGALEMENT LE FICHIER SOURCE.

8 - Autres questions non évoquées ici...

consulter la rédaction à l'adresse ci-dessous.

9 - Donnez une adresse mail et téléphone où l'on puisse vous joindre facilement au moment où l'on fait la mise en page de votre article.

\section{Contact}

Annie ANTOINE, Secrétaire de l'Association pour la publication des $A B P O$ université de Rennes 2, Département Histoire,

Place du Doyen-Henri-Le-Moal - 35043 RenNes Cedex

e-mail : annie.antoine@uhb.fr

Les manuscrits sont à envoyer à l'adresse ci-dessus

Ouvrage achevé d'imprimer

sur les presses du service reprographique

de l'Université Rennes 2 Haute-Bretagne

en octobre 2006

Imprimé en France 